\title{
Identification of abnormal lipid profiles promote cognitive decline in Alzheimer's disease spectrum via large-scale brain connectivity
}

\section{Qing Wang}

Southeast University Zhongda Hospital

\section{Feifei Zang}

Southeast University Zhongda Hospital

Cancan He

Southeast University Zhongda Hospital

\section{Zhijun Zhang}

Southeast University Zhongda Hospital

Chunming Xie ( $\nabla 101011769 @ s e u . e d u . c n$ )

Southeast University Zhongda Hospital

\section{Research}

Keywords: Alzheimer's disease, lipid, large-scale brain networks, canonical correlation analysis, functional connectivity; biomarkers

Posted Date: May 13th, 2021

DOl: https://doi.org/10.21203/rs.3.rs-496187/v1

License: (1) This work is licensed under a Creative Commons Attribution 4.0 International License. Read Full License 


\section{Abstract}

Background: Lipid metabolite dysfunction makes a substantial contribution to the clinical signs and pathophysiology of Alzheimer's disease (AD). It is unclear that the role of dyslipidemia in the promotion of neuropathological processes and brain functional impairment that subsequently facilitates the progression of $A D$.

Methods: Lipid pathway-based polygenic scores and large-scale resting-state networks (RSNs), was constructed. Together with canonical correlation analysis (CCA) and support vector machine (SVM) model, to explore the effects of lipid-related polygenic scores and blood lipoproteins on the molecular biomarkers, cognitive function, as well as large-scale RSNs. Associations between lipid-related genetic scores, serum lipoproteins, cognitive function, CSF biomarkers and RSNs were examined.

Results: Dynamic trajectory of large-scale RSNs was exhibited significantly differential connectivity within-network, one-versus-all-others-network, and pairwise between networks across the AD spectrum (ADS). Importantly, the summative effects of lipid-pathway genetic variants and lipoproteins significantly promoted the process of $\beta$-amyloid and Tau levels and cognitive decline, and preferentially targeted functional couplings within- and between RSNs in the ADS, supporting the hypothesis that abnormal lipid profiles in the AD pathogenesis via disrupting large-scale RSNs and accelerating molecular pathological processes, consequently exacerbating cognitive decline.

Conclusions: Our findings reveal the importance of lipids in the pathogenesis of $A D$ via disruption of RSNs and acceleration of molecular pathological processes, consequently exacerbating cognitive decline. These findings provide the potential lipid-associated neuroimaging biomarkers for ADS.

\section{Background}

Lipids are important components of the brain that play a critical role in the membrane formation of neuronal cells, and participate in essential physiological functions such as cellular transport, energy storage, in addition to acting to modulate transmembrane proteins and signaling molecules, promoting effective signal transduction and regulating gene expression [1-3]. In recent years, growing evidence from both animal models and humans' studies has identified abnormal lipid metabolites were associated with the molecular mechanisms underlying Alzheimer's disease (AD) pathophysiology beyond amyloid plaques and neurofibrillary tangles [4-9]. In fact, altered plasma lipid profiles have appeared to exacerbate cognitive decline, subsequently increasing the risk of the incidence of $A D$ in nondemented elderly adults $[5,7,8,10-13]$. Specifically, recent biological and neuroimaging data have indicated that the dysfunctional composition of lipid rafts, primarily located in membrane microdomains and serving as an important platform for signal processing, may contribute to AD pathophysiology $[2,8]$. Cholesterol, as a major component of lipid rafts, is thought to be involved in amyloid precursor protein (APP) processing and $\beta$-amyloid $(A \beta)$ overproduction characterized a key feature of $A D$ pathophysiology [7], while gemfibrozil, a fibric acid agent commonly used to treat hyperlipidemias in clinic, significantly reduces 
amyloid pathology and reverses memory deficits in APP-PSEN1 $\triangle E 9$ mice [14], a murine model that mimics AD-like pathology and cognitive decline. As changes of lipoprotein in the blood can be detected prior to cognitive decline, it is of considerable interest to know whether lipid pathway-based metabolites substantially contribute to AD pathophysiology [4]. However, to date, it remains unclear how lipid metabolites, cerebral spinal fluid (CSF) biomarkers, and brain function are linked or interacted with the progression of cognitive decline in preclinical or clinical AD patients.

Brain network integrity plays an instrumental role in the regulation of high-order cognitive function. Resting-state networks (RSNs), which measure temporal correlation depend on intrinsic blood oxygenation level dependent (BOLD) signals within large-scale systems and provide a powerful tool to investigate network integrity between structurally segregated and functionally specialized brain regions at the system level [15]. Importantly, the spatial-temporal evolution of RSNs has been found to be tightly associated with neural correlates of cognitive impairment observed in preclinical and clinical AD patients [16-19], including default mode network (DMN), executive control network (ECN), salience network (SAN), attention network (AN), and visuospatial network (VIS), suggesting that changes in distributed networks at a large-scale system level could predict clinical progression and neurodegeneration [16, 20, 21]. Recently, particular attention to network integrity has shifted towards investigation of molecular pathological changes invoked in intrinsic large-scale network dynamics supporting diverse cognitive function [22]. Specifically, increasing evidence has demonstrated that neural correlates of the disrupted connectivity of RSNs in cognitively healthy individuals with brain amyloidosis or AD-related genetic risk factors were similar to abnormalities observed in symptomatic AD [23-25]. As such, it may be possible that RSNs could be used as an intermediate phenotype linking downstream cognitive decline and upstream molecular cascading events underlying $A D$ pathophysiology. In reality, dysregulation of lipids is substantially associated with the disrupted architecture of RSNs and is directly involved in the molecular and cellular changes underlying AD pathophysiology [26, 27]. For example, high serum cholesterol has been associated with decreased cortical and hippocampal volumes in cholesterol-fed rabbits [28] and disrupt functional connectivity of the SAN in the non-demented elderly [26]. Increased low-density lipoprotein cholesterol (LDL-C) levels causes a detrimental effect to posterior cingulate gray matter volumes and verbal memory [29], while elevated high-density lipoprotein cholesterol (HDL-C) provides protection against hippocampal atrophy and $A D[30,31]$. From our previous work, we previously reported that the effects of the accumulation of genetic variants of cholesterol-pathway molecules produces widespread effects on cortico-subcortical-cerebellar spontaneous brain activity in amnestic mild cognitive impairment $(\mathrm{aMCl})$ patients [32]. These findings suggest that several, distinct lipidomic signatures influence brain network integrity and subsequently contribute to AD. However, it remains unclear how altered lipid metabolites impinge on the dynamic spatiotemporal patterns of RSNs as AD progresses. Indeed, in the context of lipid-centric gene and protein changes, evaluation of the potential effects of lipid abnormalities that affect dynamic brain network trajectory and CSF biomarkers, subsequently leading to cognitive decline, are beneficial in order to capture a more holistic picture of the processes of AD.

In the present study, a new approach was used that combining large-scale brain networks with canonical correlation analysis (CCA) to explore the effects of lipid metabolic disturbance on the dynamic trajectory 
of ten RSNs changes and molecular biomarkers that promote cognitive decline with progression of AD. Firstly, the relationship between lipid-centric gene variants and proteins, CSF biomarkers, and cognitive performance across the AD spectrum AD (ADS) was examined. Secondly, the dynamic trajectory of largescale network changes was identified both within- and between ten predefined RSNs from cognitive normal (CN) healthy to mild AD stage individuals. Thirdly, the potential associations between lipid-related gene variants and proteins, and the dynamic trajectory of large-scale network connectivity, CSF biomarkers, in addition to cognitive performance were explored using CCA. Finally, a support vector machine (SVM) model of machine learning was used to distinguish ADS patients from CN subjects. Taken together, these findings provided an integrated view of lipid metabolite abnormalities that affect the pathogenesis of $A D$ via mediating large-scale brain network integrity and promoting neuropathological processes and then exacerbating cognitive decline.

\section{Methods}

All data at baseline were extracted from the Alzheimer's disease Neuroimaging Initiative (ADNI) database. CCA was conducted to explore the effect of the accumulation of lipid gene- and protein-centric levels on CSF biomarkers including $\beta$-amyloid, tau, and phosphorylated tau concentrations, the dynamic trajectory of large-scale resting-state networks (RSNs), and cognitive performance across the entire ADS. Detailed methods are presented in supplementary materials.

\section{Results}

\section{Demographic, genetic and molecular biomarkers, and neuropsychological data}

There were no significant differences in age, gender, education, levels of thirty-eight serum lipid metabolites, or any candidate genotypes, except apolipoprotein E (APOE) genotypes within any groups of participants. Significant cognitive decline as signed by lower MMSE scores and higher ADAS-cog scores, gradually decreased $A \beta$ level, and increased Tau and $p$-Tau levels were identified in ADS individuals compared to the $\mathrm{CN}$. In terms of cognitive scores or CSF biomarkers, there were no significant differences between early $\mathrm{MCl}(\mathrm{EMCl})$ and late $(\mathrm{LMCl})$ groups. No genotypes deviated from the Hardy-Weinberg equilibrium with all p values above 0.05 . More details of demographic, lipid pathway-based genotypes, CSF biomarkers, and global cognition are displayed in Table 1 and Table S1 and S2. 
Table 1

Demographic data, lipid pathway-based genotypes, cerebrospinal fluid biomarkers, and global cognitive performance across the AD spectrum.

\begin{tabular}{|c|c|c|c|c|c|}
\hline & $\mathrm{CN}$ & $\mathrm{EMCl}$ & $\mathrm{LMCl}$ & $A D$ & \\
\hline Items & $(n=51)$ & $(n=26)$ & $(n=26)$ & $(n=21)$ & values \\
\hline Age (years) & $74.08 \pm 5.79$ & $70.04 \pm 6.87$ & $70.81 \pm 7.14$ & $71.81 \pm 7.77$ & 0.051 \\
\hline Gender (F/M) & $30 / 21$ & $14 / 12$ & $11 / 15$ & $9 / 12$ & $0.447 *$ \\
\hline Education (years) & $16.31 \pm 2.59$ & $15.27 \pm 2.51$ & $16.31 \pm 2.51$ & $15.14 \pm 2.76$ & 0.157 \\
\hline \multicolumn{6}{|l|}{ Multiple protective genes } \\
\hline $\begin{array}{l}\text { CLU T status (TC + } \\
\text { TT/CC) }\end{array}$ & $38 / 13$ & $17 / 9$ & $18 / 8$ & $13 / 8$ & $0.710 *$ \\
\hline $\begin{array}{l}\text { LDLR A status (AG + } \\
\text { AA/GG) }\end{array}$ & $38 / 13$ & $16 / 10$ & $15 / 11$ & $11 / 10$ & $0.240 *$ \\
\hline $\begin{array}{l}\text { LRP1 T status }(\mathrm{TC}+ \\
\text { TT/CC) }\end{array}$ & $15 / 36$ & $8 / 18$ & $5 / 21$ & $10 / 11$ & $0.214^{\star}$ \\
\hline $\begin{array}{l}\text { PICALM A status }(A G+ \\
\text { AA/GG) }\end{array}$ & $27 / 24$ & $15 / 11$ & $16 / 10$ & $11 / 10$ & $0.884^{*}$ \\
\hline \multicolumn{6}{|l|}{ Multiple risk genes } \\
\hline APOE $\varepsilon 4$ status (+/-) & $15 / 36$ & $14 / 12$ & $12 / 14$ & $15 / 6$ & $0.008 *$ \\
\hline $\begin{array}{l}\text { SORL1 G status (TG + } \\
\text { GG/TT) }\end{array}$ & $19 / 32$ & $12 / 14$ & $12 / 14$ & $6 / 15$ & $0.548 *$ \\
\hline $\begin{array}{l}\text { CETP A status (AG + } \\
\text { AA/GG) }\end{array}$ & $46 / 5$ & $24 / 2$ & $23 / 3$ & $19 / 2$ & $0.974^{\star}$ \\
\hline $\begin{array}{l}\text { ABCA1 G status }(A G+ \\
\text { GG/AA) }\end{array}$ & $45 / 6$ & $25 / 1$ & $21 / 5$ & $19 / 2$ & $0.369 *$ \\
\hline $\begin{array}{l}\text { BIN1 C status }(\mathrm{TC}+ \\
\mathrm{CC} / \mathrm{TT})\end{array}$ & $27 / 24$ & $17 / 9$ & $15 / 11$ & $11 / 10$ & $0.739 *$ \\
\hline \multicolumn{6}{|l|}{$\begin{array}{l}\text { Cerebrospinal fluid } \\
\text { biomarkers }\end{array}$} \\
\hline$A \beta(p g / m l)$ & $\begin{array}{l}192.79 \pm \\
50.17^{\mathrm{bc}}\end{array}$ & $\begin{array}{l}183.61 \pm \\
50.66^{d}\end{array}$ & $\begin{array}{l}168.77 \pm \\
50.80\end{array}$ & $\begin{array}{l}140.40 \pm \\
43.59\end{array}$ & 0.001 \\
\hline Tau $(\mathrm{pg} / \mathrm{ml})$ & $\begin{array}{l}68.53 \pm \\
34.14^{c}\end{array}$ & $\begin{array}{l}79.32 \pm \\
51.89^{d}\end{array}$ & $\begin{array}{l}86.01 \pm \\
52.19^{\mathrm{e}}\end{array}$ & $\begin{array}{l}129.29 \pm \\
61.42\end{array}$ & $<.001$ \\
\hline pTau (pg/ml) & $\begin{array}{l}34.18 \pm \\
16.58^{\mathrm{bc}}\end{array}$ & $\begin{array}{l}39.60 \pm \\
24.73^{d}\end{array}$ & $\begin{array}{l}48.42 \pm \\
33.50\end{array}$ & $\begin{array}{l}55.23 \pm \\
26.13\end{array}$ & 0.005 \\
\hline
\end{tabular}




\begin{tabular}{|c|c|c|c|c|c|}
\hline & $\mathrm{CN}$ & EMCI & LMCI & $A D$ & \multirow{2}{*}{$\begin{array}{l}\mathrm{P} \\
\text { values }\end{array}$} \\
\hline Items & $(n=51)$ & $(n=26)$ & $(n=26)$ & $(n=21)$ & \\
\hline \multicolumn{6}{|c|}{$\begin{array}{l}\text { Global cognitive } \\
\text { performance }\end{array}$} \\
\hline MMSE & $\begin{array}{l}28.84 \pm \\
1.16^{\mathrm{abc}}\end{array}$ & $\begin{array}{l}27.92 \pm \\
2.13^{d}\end{array}$ & $\begin{array}{l}27.73 \pm \\
1.54^{\mathrm{e}}\end{array}$ & $22.67 \pm 2.50$ & $\begin{array}{l}< \\
0.001\end{array}$ \\
\hline ADAS-Cog & $\begin{array}{l}10.76 \pm \\
6.53^{\mathrm{abc}}\end{array}$ & $\begin{array}{l}14.19 \pm \\
6.58^{d}\end{array}$ & $\begin{array}{l}16.96 \pm \\
5.32^{\mathrm{e}}\end{array}$ & $35.81 \pm 8.99$ & $\begin{array}{l}<.001 \\
0.01\end{array}$ \\
\hline \multicolumn{6}{|c|}{ 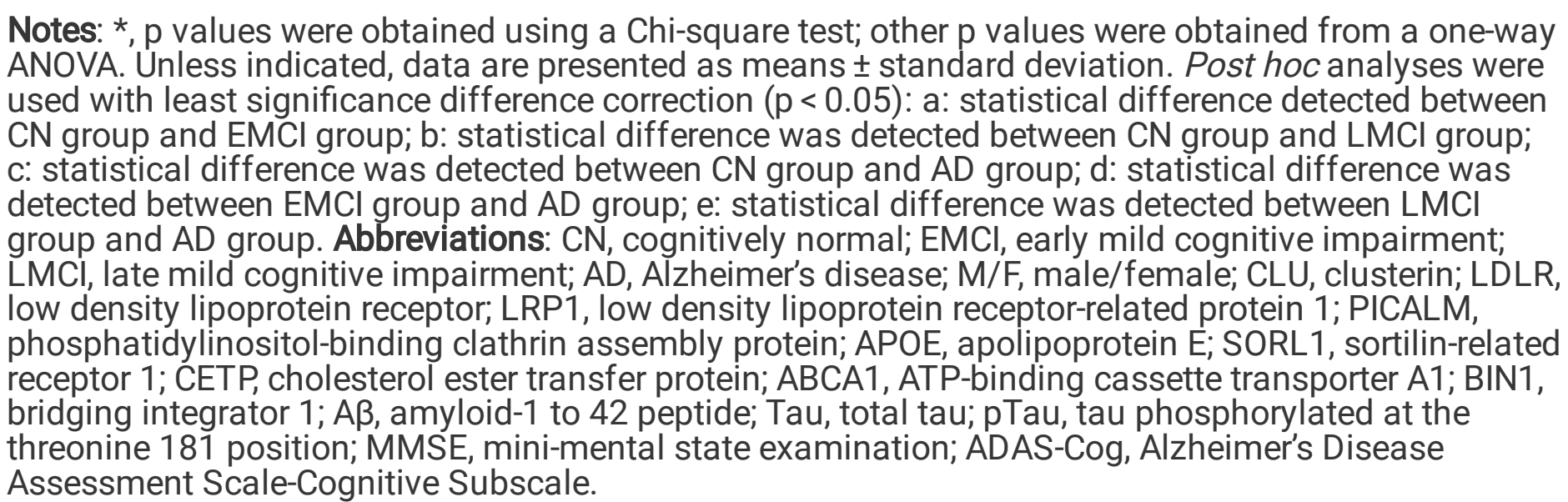 } \\
\hline
\end{tabular}

\section{Relationships among polygenic scores, lipid metabolites, CSF biomarkers and cognitive performance}

First, binomial nonlinear connections were discovered between five cholesterol metabolism related biomarkers in the serum and ADAS-cog score, including serum total cholesterol (SERUM_C), esterified cholesterol (ESTC), free cholesterol (FREEC), phosphatidylcholine (PC), and sphingomyelins (SM). Besides, TOTPG (total phosphoglycerides), total choline (TOTCHO), and S_HDL_P (total lipids, phospholipids, total cholesterol, cholesterol esters, free cholesterol and triglycerides in small HDL particles) levels represented a correlation trend with ADAS-cog or MMSE scores. Then, three cholesterol metabolism related markers including SERUM_C, ESTC and SM were also linearly correlated with Tau but not $A \beta$ and $p$-Tau levels of CSF in the ADS. In addition, linear regression between polygenic scores and CSF biomarkers disclosed that genetic risk scores (GRS) was significantly correlated with $A \beta, T a u$, and even p-Tau levels, while genetic protective score (GPS) was not correlated with any of CSF biomarkers. It is interesting that relative risk scores (RRS = GRS - GPS) was significantly influenced the A $\beta$, Tau but not $p$-Tau levels in the ADS. Of note, the correlations between GRS_n (GRS without APOE), RRS_n (RRS without APOE) and CSF markers were not found, so the graphs were not presented here. All the corresponding graphs above were plotted in Fig. 1. Meanwhile, regression analyses revealed that CSF biomarkers (including $A \beta$, Tau and $p$-Tau) could significantly impact global cognitive performance in a nonlinear manner (Fig. S1). 


\section{Dynamic trajectory of large-scale brain network roles across the AD spectrum}

To explore the dynamic trajectory both within- and between RSNs in ADS patients, pairwise functional connections (correlations) were extracted within- and between ten predefined large-scale functional brain networks: auditory network (AUD), cingulo-opercular network (CON), dorsal attention network (DAN), DMN, fronto-parietal network (FPN), SAN, sensory network (SMN), subcortical network (SUB), ventral attention network (VAN), and visual network (VIS), as derived from the brain atlas of Power et al. [33]. By mapping the group mean within-network connectivity (WNC) and between-network connectivity (BNC) to a 2D parameter space, the mean functional role of 10 RSNs was qualitatively described across the ADS (Fig. 2A-D). From the means of individual WNC and BNC values (depicted by horizontal and vertical dotted lines in Fig. 2F, detailed information provided in Supplemental Materials), the RSNs from the CN group were consequently classified into four network roles: cohesive connector (SAN, DAN, SMN, and SUB), cohesive provincial (VIS), incohesive connector (AUD, FPN and CON), or incohesive provincial (DMN and VAN) (Fig. 2A). In addition to DMN and VAN, which exhibited both weaker cohesive connector and cohesive provincial roles, the other eight networks in the ADS represented divergent network roles compared to those of the CN group. Specifically, SAN, DAN, and AUD represented incohesive provincial and connector networks in patients with $\mathrm{EMCl}, \mathrm{LMCl}$ and $\mathrm{AD}$, respectively, the converse of that observed in $\mathrm{CN}$ subjects (Fig. 2A-D). The graphs visually demonstrated how the network roles of these large-scale RSNs dynamically changed with severity of disease (Fig. 2E). Interestingly, the strengths of WNC and BNC exhibited a dynamically weakened trend, except for SUB, as disease progressed through the ADS, indicating that spatiotemporal patterns of large-scale RSNs represent a rebounding network mode rather than cascading network failure, as described previously [16].

\section{Group-level Comparison Of Network Connectivity In Ad Spectrum Individuals}

We next tested differences in WNC and BNC in terms of large-scale RSNs among the four groups. Firstly, we obtained distinctive WNC and BNC matrices of the 10 RSNs for the four groups (Fig. 2G). Clearly, five RSNs (DAN, FPN, SAN, VAN, and VIS) exhibited significantly differential WNC among the disease spectrum (Fig. 2H). Although VAN and DMN were found to be incohesive provincial networks in four groups (Fig. 2A-D), VAN exhibited significantly lower WNC and BNC across the ADS (Fig. 2A-D). Similarly, five RSNs, including DAN, SAN, SMN, CON, and AUD, were found to have incohesive connector roles and provincial networks that had lower connectivity in the ADS relative to $\mathrm{CN}$ subjects. It was noted that the FPN shifted from an incohesive connector to incohesive provincial network while the VIS changed from cohesive connector to a cohesive provincial network, both representing lower connectivity in the ADS relative to $\mathrm{CN}$ subjects. In addition, the SUB displayed more cohesive connector and cohesive provincial networking, having the greatest connectivity in ADS individuals compared with $\mathrm{CN}$ subjects. Furthermore, ADS patients also showed significantly differential one-versus-all-other-network connectivity in the DAN, FPN, SAN, VAN, and VIS networks compared with controls (Fig. 2H).

In addition, pairwise BNC was calculated as the mean connectivity between each pair of RSN. Connectivity profiles of patients with $\mathrm{EMCl}, \mathrm{LMCl}$ and $\mathrm{AD}$ were compared with controls. Figure $2 \mathrm{H}$ demonstrates that pairwise BNC was significantly different for ADS patients in the following pairs: AUD- 
VAN, AUD-VIS, CON-DAN, CON-VAN, DMN-FPN, DMN-VAN, DAN-FPN, DAN-SMN, FPN-VAN, SUB-VIS and VAN-VIS. Furthermore, post hoc analysis indicated that the source of the significant differences in these pairwise BNC groups was at the large-scale network level. Specifically, ADS patients were characterized by continuous hypoconnectivity and dynamically hyperconnected links among the ten predefined RSNs as disease progressed (Fig. $2 \mathrm{I}$ and $2 \mathrm{~J}$ ). These original alterations of large-scale networks may initially reproduce those spatiotemporal pattern discrepancies, accounting for proposed molecular pathophysiological mechanisms at the distributed network level.

\section{Correlation patterns of large-scale network connectivity with CSF biomarkers and cognitive performance in the $A D$ spectrum}

To explore the potential relationship between the dynamic trajectory of connectivity of the RSNs and CSF biomarkers or cognitive performance, a new method of combination network analysis and CCA was utilized. Recent studies have demonstrated that CCA, a powerful multivariate approach that seeks to identify clusters of maximal correlation between two groups of variables, can detect associations between structural or functional connectivity and other phenotypic measures [33, 34]. Using this method, we demonstrated that large-scale brain network abnormalities were significantly correlated with phenotypic variations and molecular biomarkers in the ADS. In the first step, univariate correlation was used to test the composition of the clinical CCA mode with each of the two clinical variables (MMSE and ADAS-cog). We observed that clinical CCA mode was highly correlated with MMSE score $(r=0.78, p<$ $0.0001)$ and ADAS-cog score $(r=0.78, p<0.0001)$ (Fig. 3A). Similarly, we identified that CSF CCA mode was highly correlated with levels of Tau $(r=0.72, p<0.0001)$ and pTau $(r=0.68, p<0.0001)$, and moderately correlated with $A \beta 42(r=0.55, p<0.0001)$ (Fig. 3B). As shown in Fig. 3C, the network CCA mode was significantly correlated with 55 original network variables (Table S4). In total, the CCA model of network was significantly correlated with clinical variate CCA (Fig. 3D, $r=0.93, p<0.0001$ ) and CSF CCA (Fig. 3E, $r=0.95, p<0.0001$ ) modes, respectively.

\section{Correlation patterns of lipid pathway-based genetic variants and lipoproteins with large-scale network connectivity in AD spectrum patients}

Similarly, we also performed CCA to ascertain the association of brain network connectivity measures with accumulated lipid-related genetic scores and lipoproteins in the blood of ADS patients. We firstly tested univariate correlations for each of the 3 gene variables and 38 serum lipid variables in order to better understand the composition of gene CCA and serum lipid CCA modes. We found that first gene CCA mode was highly correlated with GRS $(r=1, p<0.0001)$, GPS $(r=1, p<0.0001)$ and RRS $(r=1, p<$ 0.0001) (Fig. 4A). As shown in Fig. 4B, serum lipid CCA mode was significantly correlated with all 38 original serum lipid variables (Table S5). The results of third pair CCA mode of network and gene variate were again highly significant (Fig. 4C, $r=0.97, p<0.0001$ ), as was fourth pair CCA mode of network and serum lipid variable (Fig. 4D, $r=0.82, p<0.0001$ ).

In order to determine the potential for APOE $\varepsilon 4$ genotype to alter the association between lipid metabolism-related genes and dynamic changes in RSNs, we constructed a second gene CCA mode and 
found that second order gene CCA mode was highly correlated with GRS_n $(r=1, P<0.0001)$ and RRS_n scores $(r=1, P<0.0001)$ after removing the APOE $\varepsilon 4$ genotype (Fig. S2A). Fifth pair CCA mode of the network and three gene score variables where removed APOE $\varepsilon 4$ OR values was also significantly correlated (Fig. S2C, $r=0.94, p<0.0001$ ).

\section{Post hoc analysis revealed the potential of distinctive lipid-related genetic scores and lipoproteins on large-scale network connectivity, CSF biomarkers, and cognitive performance}

To determine the direction and magnitude of these associations between network CCA mode and a single variate of a clinical indicator, we conducted post hoc correlation analysis. As illustrated in Fig. 5A, nineteen lipoproteins were mostly associated with increased network connectivity and seven with decreased network connectivity within- and between- ten predefined RSNs. Furthermore, GRS was positively associated with increased network connectivity within the SUB and negatively associated with altered network connectivity between CON-VAN, DAN-VAN, FPN-VAN, SAN-VAN, SUB-VAN, VAN-VIS, and AUD-VAN, while GPS was negatively associated with decreased network connectivity between the FPN and SUB. Similarly, RRS was positively associated with decreased network connectivity between DMNSUB, DAN-SUB, FPN-SUB, CON-DMN, AUD-CON, and negatively associated with decreased network connectivity between AUD-VAN, and DAN-VAN, whereas RRS was associated with increased network connectivity within the SUB. In addition, MMSE was negatively correlated with decreased network connectivity between DMN and SAN, while ADAS-cog and Tau were mostly associated with increased network connectivity between SAN-SUB, and DMN-SAN, as shown in Fig. 5B. It is noteworthy that GRS was only associated with decreased network connectivity between SAN-VAN after removing the effects of the APOE $\varepsilon 4$ genotype (Fig. S3). Detailed information for these correlation coefficients ( $r$ ) and $p$ values are described in Table $\mathbf{S 6 .}$

\section{SVM analyses identified potential lipid-associated imaging biomarker for AD spectrum}

After post-hoc analysis, we performed correlation analysis, and found that there were six functional connections significantly correlated to all nineteen lipoproteins and sixteen functional connections related to all three gene scores (Table S7). Then, these twenty-two features were used for classification. The SVM model revealed that the lipid-associated twenty-two functional connections represented higher capacity to discriminate disease spectrum (AUC between 0.82-0.92), as shown in Fig. 6.

\section{Discussion}

This is the first study focusing on the potential of lipid-related genes and proteins to influence the dynamic trajectory of large-scale RSNs, CSF biomarkers, and cognitive decline in ADS patients using a CCA approach. The present study shed mechanistic light on the role of disturbance in lipid metabolites to promote large-scale RSNs disruption and acceleration of CSF biomarker deposition in mediating cognitive decline in ADS individuals. These findings provide novel insight for uncovering the neural link between lipid metabolites and cognitive decline at a large-scale network level and expanding our understanding of the mechanisms underlying AD pathophysiology. 
Although it is not well-established that potential relationships between lipid metabolites and AD exist, the majority of studies have reported that abnormal lipid metabolites apparently increased the risk of cognitive decline and substantially contribute to the development and progression of $A D[3,5,35,36]$. Recently, a meta-analysis reported that high midlife total serum cholesterol significantly increases the risk of late-life AD, and may correlate with the onset of AD pathology [37]. A prospective study with a largecohort sample in which 22,623 participants were recruited established that the concentration of cholesterol esters relative to total lipids in large HDL and the total cholesterol to total lipid ratio in very large VLDL significantly increased the risk of incidence of dementia [5]. We also found that lipid metabolites, including genes and lipoproteins were markedly associated with CSF biomarkers and cognitive impairment, also supporting the hypothesis that lipid metabolic dysfunction substantially contributes to $A D$ pathophysiology via interference through progressive neuropathological changes of CSF biomarkers and declining cognitive function across the entire ADS.

Disrupted network integrity, including abnormal structural and functional network connectivity, was preferentially targeted by specific genetic variants or molecular pathology in preclinical $A D$, or mapped the clinical phenotype with disease progression and supported the recent description of the theoretical framework and empirical evidence of $A D[22,38]$. As such, brain network integrity emerged as potential intermediate biomarkers to bridge upstream determinants (gene and molecular pathology) and downstream effects (clinical phenotypes) [21, 22]. However, the complicated association that the dynamic spatiotemporal patterns of brain network integrity linking molecular pathology and cognitive decline in ADS individuals remains largely unclear. According to cascading network failure theory, distinct DMN subsystems representing differential spatiotemporal evolution correspond with the AD pathophysiological response, and differentially affected by $A D$ pathological biomarkers including $A \beta$ deposition and tau tangles, subsequently leading to stereotypic network-based cognitive decline in ADS patients $[16,20]$. This study firstly described the progressive changes in spatiotemporal network patterns within the DMN system in ADS patients. We then further identified changes in dynamic trajectory withinand between networks reflected by the active capability of network inner cohesion and connectors beyond the DMN across the entire ADS. More attention should be focused on whether such changes in dynamic trajectory in large-scale RSNs are cascading failure or not. In contrast, a proportion of the networks represented enhanced network inner cohesion or exhibited network connector roles as the disease progressed. Compelling evidence has been reported that a gradual decrease in connectivity of RSNs is associated with amyloid deposition that accelerates disease progression, while the commonly observed increase in connectivity of RSNs also found in preclinical and prodromal AD patients has been interpreted as a compensatory phenomenon supporting better performance on cognitive tasks $[39,40]$. However, this enhanced network connectivity is the consequence of transient compensation to network disruption or an adaptive response to $A D$ pathophysiological processes that still require identification through additional study.

Importantly, the dynamic changes in large-scale networks over the course of disease that were also observed were significantly affected by lipid-related genetic variants and lipoproteins, CSF biomarkers, and cognitive function, confirming the biological nature of the predictable correlation with network 
connectivity by linking upstream molecular pathology and downstream clinical phenotype to the preclinical stage of AD. Furthermore, post hoc analysis was performed to trace the source of these system-level correlations and identified that distinctive connectivity within- and between networks was specifically related to the effects of accumulated lipid-related genetic variants or lipoproteins, neuropathological biomarkers, in addition to cognitive decline. Due to changes in lipids often prior to molecular pathology and cognitive decline, we hypothesized that compromised large-scale networks and CSF biomarkers may mediate the effects of lipid metabolites on cognitive decline with progression of AD. Previously, structural atrophy or functional decoupling of RSNs were, at least partly, ascribed to abnormalities in lipid metabolites which suggested that lipid metabolites may be a vulnerable molecular substrate of large-scale RSNs [26, 32]. More importantly, disturbed lipid metabolites and dynamic brain network changes occurred prior to measurable amyloid deposition and tau tangles related to ageing [16], while lipid pathway genetic variants, including APOE 44 genotype and lipoproteins markedly enhanced the disruption of brain network architecture in preclinical $A D$ patients $[32,41]$ and even in the cognitively normal elderly $[42,43]$. In addition, carriers of the APOE\&4 allele, the strongest risk factor for sporadic lateonset $A D$, represented a specific phenotype in which the relationship with brain networks preceded any measurable systems or molecular level changes in cognitively normal subjects [44-46]. Furthermore, cholesterol-related genetic risk scores were associated with hypometabolism in AD-affected brain regions, even when controlling for the effects of APOE $\varepsilon 4$ gene dose [47]. In this regard, we putatively identified that the dynamic trajectory changes of large-scale networks observed in this study may be induced as a consequence of a lipid-driven pathological interaction with $A \beta$ abnormal deposition and tau-related neurofibrillary tangles that then promote cognitive decline to dementia. However, a greater level of evidence is required to elucidate the causal relationship between lipid-related genetic variants, lipoproteins and dynamic large-scale network changes in ADS individuals.

Another interesting finding of the present study was the SVM classifier model, This SVM classifier achieved a relatively high performance. This also implies that a significantly important role of lipid metabolism in the development of AD. Lipid associated neuroimaging biomarkers would serve as a good potential biomarker for ADS diagnoses.

\section{Limitations}

Two limitations of this study should be noted. Firstly, the 38 lipid-related genetic variants and lipoproteins selected in this multimodal cross-sectional study may underestimate the potential of lipid metabolites for the early detection and diagnosis of AD. Lipidomics approaches should be considered in order to explore the pathogenesis of $A D$, because this provides a new tool to investigate the association between bloodbased genetic variants or changes in lipoproteins in the serum or plasma and the pathological mechanisms of CNS disorders. Secondly, longitudinal studies should be performed to explore the potential biomarkers of lipid metabolites in AD pathophysiology, validate the links between changes in lipids and neuropathology, and determine the causal contributions of lipid metabolite disturbance and disrupted network integrity, in addition to cognitive decline in ADS patients. 


\section{Conclusions}

To sum, we demonstrate that dynamic trajectory of large-scale RSNs represents a rebounding mode rather than a cascading failure mode with disease progression in the ADS, and more importantly, abnormal lipid metabolite changes may induce the disruption of large-scale RSNs and CSF biomarker deposition, which then promote cognitive impairment in preclinical and clinical AD. These findings provide new evidence in which an effective strategy for early prevention or disease-modifying therapy that targets the metabolites of lipid-related genetic variants or lipoproteins for late-onset $A D$, which would significantly improve our understanding of the mechanisms underlying the association of lipid molecules and $A D$ pathophysiology.

\section{List Of Abbreviations}

$\mathrm{CN}$, cognitively normal; $\mathrm{EMCl}$, early mild cognitive impairment; $\mathrm{LMCl}$, late mild cognitive impairment; $A D$, Alzheimer's disease; MMSE, mini-mental state examination; ADAS-Cog, Alzheimer's Disease Assessment Scale-Cognitive Subscale; GPS, genetic protective score; GRS, genetic risk score; RRS, relative risk score; CCA, canonical correlation analysis; AUD, the auditory network; CON, the cingulo-opercular network; DAN, the dorsal attention network; DMN, the default mode network; FPN, the fronto-parietal network; SAN, the salience network; SMN, the sensory network; SUB, the subcortical network; VAN, the ventral attention network; VIS, the visual network; $A \beta$, amyloid 1 to 42 peptide; Tau, total tau; $p T a u$, tau phosphorylated at the threonine 181 position; SERUM_C, serum total cholesterol; ESTC, esterified cholesterol; FREEC, free cholesterol; PC, phosphatidylcholine; SM, sphingomyelins; TOTCHO, total cholines.

\section{Declarations}

\section{Ethical Approval and Consent to participate}

Ethical approval was obtained by the ADNI investigators (http://www.adniinfo.org/pdfs/adni_protocol_9_19_08.pdf). All Institutional Review Boards of all participating sites at their respective institutions approved the study. All ADNI participants provided written informed consent before the start of the study.

\section{Consent for publication}

Not applicable

\section{Availability of data and materials}

The datasets used and/or analyzed during the current study are available from the corresponding author on reasonable request.

\section{Competing interests}


The authors declare that they have no competing interests.

\section{Funding}

This research was supported by the National Key Projects for Research and Development Program of China [2016YFC1305800, 2016YFC1305802, CMX], the National Natural Science Foundation of China [82071204, 81671256, 81871069, CMX], the Key Project for Research and Development Program of Jiangsu Province [BE2018741], the Key Projects of Jiangsu Commission of Health [ZDB2020008, CMX], and the Nanjing International Joint Research and Development Project [201715013].

\section{Authors' contributions}

All authors have made substantial intellectual contributions to this manuscript in one or more of the following areas: design or conceptualization of the study, analysis or interpretation of the data, or drafting or revision of the manuscript. Dr. Xie and Prof. Zhang design this study. Ms. Wang and Ms. Zang conducted the data preparation and statistical analysis. Ms. Wang and Ms. He did the data preparation. Dr. Xie guide to do data analysis. All authors have given final approval of this manuscript.

\section{Acknowledgments}

Data collection and sharing for this project was funded by the ADNI (National Institutes of Health Grant No. U01 AG024904). Data used in preparation of this article were obtained from the ADNI databases (www.loni.usc.edu). As such, the investigators within the ADNI contributed to the design and implementation of $A D N I$ and/or provided data but did not participate in analysis or writing of this report. A complete listing of ADNI-D and ADNI investigators can be found at: http://adni.loni.usc.edu/wpcontent/uploads/how_to_apply/ ADNI_Acknowledgement_List.pdf.

\section{Authors' information}

${ }^{1}$ Department of Neurology, Affiliated ZhongDa Hospital, School of Medicine, Southeast University, Nanjing, Jiangsu, China, 210009. ${ }^{2}$ Neuropsychiatric Institute, Affiliated ZhongDa Hospital, Southeast University, Nanjing, Jiangsu, China, 210009. ${ }^{3}$ The Key Laboratory of Developmental Genes and Human Disease, Southeast University, Nanjing, Jiangsu, China, 210009. ${ }^{4}$ The data used in preparing this article were obtained from the Alzheimer's disease Neuroimaging Initiative (ADNI) database (http://www.loni.ucla.edu/ADNI). Investigators within the database contributed to the design and implementation of the ADNI and/or provided data but did not participate in the analysis or write this report. Please see the complete listing of ADNI investigators in the Supplementary Materials.

\section{References}

1. Brugger B. Lipidomics: analysis of the lipid composition of cells and subcellular organelles by electrospray ionization mass spectrometry. Annu Rev Biochem. 2014;83:79-98. 
2. Mesa-Herrera F, Taoro-Gonzalez L, Valdes-Baizabal C, Diaz M, Marin R. Lipid and Lipid Raft Alteration in Aging and Neurodegenerative Diseases: A Window for the Development of New Biomarkers. Int J Mol Sci. 2019;20(15).

3. Wong MW, Braidy N, Poljak A, Pickford R, Thambisetty M, Sachdev PS. Dysregulation of lipids in Alzheimer's disease and their role as potential biomarkers. Alzheimers Dement. 2017;13(7):810-27.

4. Stukas S, Robert J, Wellington CL. High-density lipoproteins and cerebrovascular integrity in Alzheimer's disease. Cell Metab. 2014;19(4):574-91.

5. Tynkkynen J, Chouraki V, van der Lee SJ, Hernesniemi J, Yang Q, Li S, et al. Association of branchedchain amino acids and other circulating metabolites with risk of incident dementia and Alzheimer's disease: A prospective study in eight cohorts. Alzheimers Dement. 2018;14(6):723-33.

6. Kunkle BW, Grenier-Boley B, Sims R, Bis JC, Damotte V, Naj AC, et al. Genetic meta-analysis of diagnosed Alzheimer's disease identifies new risk loci and implicates Abeta, tau, immunity and lipid processing. Nat Genet. 2019;51(3):414-30.

7. Di Paolo G, Kim TW. Linking lipids to Alzheimer's disease: cholesterol and beyond. Nat Rev Neurosci. 2011;12(5):284-96.

8. Proitsi P, Kim M, Whiley L, Simmons A, Sattlecker M, Velayudhan L, et al. Association of blood lipids with Alzheimer's disease: A comprehensive lipidomics analysis. Alzheimers Dement. 2017;13(2):14051.

9. Sepulcre J, Grothe MJ, d'Oleire Uquillas F, Ortiz-Teran L, Diez I, Yang HS, et al. Neurogenetic contributions to amyloid beta and tau spreading in the human cortex. Nat Med. 2018;24(12):1910-8.

10. Solomon A, Kareholt I, Ngandu T, Wolozin B, Macdonald SW, Winblad B, et al. Serum total cholesterol, statins and cognition in non-demented elderly. Neurobiol Aging. 2009;30(6):1006-9.

11. Zarrouk A, Debbabi M, Bezine M, Karym EM, Badreddine A, Rouaud O, et al. Lipid Biomarkers in Alzheimer's Disease. Curr Alzheimer Res. 2018;15(4):303-12.

12. Broce IJ, Tan CH, Fan CC, Jansen I, Savage JE, Witoelar A, et al. Dissecting the genetic relationship between cardiovascular risk factors and Alzheimer's disease. Acta Neuropathol. 2019;137(2):209-26.

13. Sepulcre J, Grothe MJ, d'Oleire Uquillas F, Ortiz-Teran L, Diez I, Yang HS, et al. Neurogenetic contributions to amyloid beta and tau spreading in the human cortex. Nat Med. 2018;24(12):1910-8.

14. Luo R, Su LY, Li G, Yang J, Liu Q, Yang LX, et al. Activation of PPARA-mediated autophagy reduces Alzheimer disease-like pathology and cognitive decline in a murine model. Autophagy. 2020;16(1):5269.

15. Xie C, Goveas J, Wu Z, Li W, Chen G, Franczak M, et al. Neural basis of the association between depressive symptoms and memory deficits in nondemented subjects: resting-state fMRI study. Hum Brain Mapp. 2012;33(6):1352-63.

16. Jones DT, Knopman DS, Gunter JL, Graff-Radford J, Vemuri P, Boeve BF, et al. Cascading network failure across the Alzheimer's disease spectrum. Brain. 2016;139(Pt 2):547-62. 
17. Palop JJ, Mucke L. Network abnormalities and interneuron dysfunction in Alzheimer disease. Nature Reviews Neuroscience. 2016;17(12):777-92.

18. Xie C, Bai F, Yu H, Shi Y, Yuan Y, Chen G, et al. Abnormal insula functional network is associated with episodic memory decline in amnestic mild cognitive impairment. Neuroimage. 2012;63(1):320-7.

19. Greicius MD, Krasnow B, Reiss AL, Menon V. Functional connectivity in the resting brain: A network analysis of the default mode hypothesis. Proceedings of the National Academy of Sciences of the United States of America. 2003;100(1):253-8.

20. Jones DT, Graff-Radford J, Lowe VJ, Wiste HJ, Gunter JL, Senjem ML, et al. Tau, amyloid, and cascading network failure across the Alzheimer's disease spectrum. Cortex. 2017;97:143-59.

21. Teipel S, Drzezga A, Grothe MJ, Barthel H, Chetelat G, Schuff N, et al. Multimodal imaging in Alzheimer's disease: validity and usefulness for early detection. Lancet Neurol. 2015;14(10):1037-53.

22. Pievani M, de Haan W, Wu T, Seeley WW, Frisoni GB. Functional network disruption in the degenerative dementias. Lancet Neurol. 2011;10(9):829-43.

23. Sperling RA, Laviolette PS, O'Keefe K, O'Brien J, Rentz DM, Pihlajamaki M, et al. Amyloid deposition is associated with impaired default network function in older persons without dementia. Neuron. 2009;63(2):178-88.

24. Hanseeuw BJ, Betensky RA, Jacobs HIL, Schultz AP, Sepulcre J, Becker JA, et al. Association of Amyloid and Tau With Cognition in Preclinical Alzheimer Disease: A Longitudinal Study. JAMA Neurol. 2019.

25. Wang L, Brier MR, Snyder AZ, Thomas JB, Fagan AM, Xiong C, et al. Cerebrospinal fluid Abeta42, phosphorylated Tau181, and resting-state functional connectivity. JAMA Neurol. 2013;70(10):1242-8.

26. Zhang T, Li H, Zhang J, Li X, Qi D, Wang N, et al. Impacts of High Serum Total Cholesterol Level on Brain Functional Connectivity in Non-Demented Elderly. J Alzheimers Dis. 2016;50(2):455-63.

27. de Bruijn RF, Akoudad S, Cremers LG, Hofman A, Niessen WJ, van der Lugt A, et al. Determinants, MRI correlates, and prognosis of mild cognitive impairment: the Rotterdam Study. J Alzheimers Dis. 2014;42 Suppl 3:S239-49.

28. Jin P, Pan Y, Pan Z, Xu J, Lin M, Sun Z, et al. Alzheimer-like brain metabolic and structural features in cholesterol-fed rabbit detected by magnetic resonance imaging. Lipids Health Dis. 2018;17(1):61.

29. Chung CP, Chou KH, Peng LN, Liu LK, Lee WJ, Chen LK, et al. Associations between low circulatory low-density lipoprotein cholesterol level and brain health in non-stroke non-demented subjects. Neuroimage. 2018;181:627-34.

30. Armstrong NM, An Y, Beason-Held L, Doshi J, Erus G, Ferrucci L, et al. Predictors of neurodegeneration differ between cognitively normal and subsequently impaired older adults. Neurobiol Aging. 2019;75:178-86.

31. Wang H, Eckel RH. What are lipoproteins doing in the brain? Trends Endocrinol Metab. 2014;25(1):814. 
32. Bai F, Yuan Y, Shi Y, Zhang Z. Multiple genetic imaging study of the association between cholesterol metabolism and brain functional alterations in individuals with risk factors for Alzheimer's disease. Oncotarget. 2016;7(13):15315-28.

33. Power JD, Cohen AL, Nelson SM, Wig GS, Barnes KA, Church JA, et al. Functional network organization of the human brain. Neuron. 2011;72(4):665-78.

34. Smith SM, Nichols TE, Vidaurre D, Winkler AM, Behrens TE, Glasser MF, et al. A positive-negative mode of population covariation links brain connectivity, demographics and behavior. Nat Neurosci. 2015;18(11):1565-7.

35. Barupal DK, Baillie R, Fan S, Saykin AJ, Meikle PJ, Arnold M, et al. Sets of coregulated serum lipids are associated with Alzheimer's disease pathophysiology. Alzheimers Dement (Amst). 2019;11:61927.

36. Bai B, Wang X, Li Y, Chen PC, Yu K, Dey KK, et al. Deep Multilayer Brain Proteomics Identifies Molecular Networks in Alzheimer's Disease Progression. Billoski, TV (1992) Introduction to Paleontology, 6th edn (New York: Institutional Press). 2020;Online ahead of print.

37. Anstey KJ, Ashby-Mitchell K, Peters R. Updating the Evidence on the Association between Serum Cholesterol and Risk of Late-Life Dementia: Review and Meta-Analysis. J Alzheimers Dis. 2017;56(1):215-28.

38. Pievani M, Filippini N, van den Heuvel MP, Cappa SF, Frisoni GB. Brain connectivity in neurodegenerative diseases-from phenotype to proteinopathy. Nat Rev Neurol. 2014;10(11):620-33.

39. Elman JA, Oh H, Madison CM, Baker SL, Vogel JW, Marks SM, et al. Neural compensation in older people with brain amyloid-beta deposition. Nat Neurosci. 2014;17(10):1316-8.

40. Jagust WJ, Mormino EC. Lifespan brain activity, beta-amyloid, and Alzheimer's disease. Trends Cogn Sci. 2011;15(11):520-6.

41. Zhu Y, Gong L, He C, Wang Q, Ren Q, Xie C, et al. Default Mode Network Connectivity Moderates the Relationship Between the APOE Genotype and Cognition and Individualizes Identification Across the Alzheimer's Disease Spectrum. J Alzheimers Dis. 2019;70(3):843-60.

42. Chen Y, Chen K, Zhang J, Li X, Shu N, Wang J, et al. Disrupted functional and structural networks in cognitively normal elderly subjects with the APOE varepsilon4 allele. Neuropsychopharmacology. 2015;40(5):1181-91.

43. Cavedo E, Chiesa PA, Houot M, Ferretti MT, Grothe MJ, Teipel SJ, et al. Sex differences in functional and molecular neuroimaging biomarkers of Alzheimer's disease in cognitively normal older adults with subjective memory complaints. Alzheimers Dement. 2018;14(9):1204-15.

44. Song H, Long H, Zuo X, Yu C, Liu B, Wang Z, et al. APOE Effects on Default Mode Network in Chinese Cognitive Normal Elderly: Relationship with Clinical Cognitive Performance. PLoS One. 2015;10(7):e0133179.

45. Trachtenberg AJ, Filippini N, Ebmeier KP, Smith SM, Karpe F, Mackay CE. The effects of APOE on the functional architecture of the resting brain. Neurolmage. 2012;59(1):565-72. 
46. Goveas JS, Xie C, Chen G, Li W, Ward BD, Franczak MB, et al. Functional network endophenotypes unravel the effects of apolipoprotein e epsilon 4 in middle-aged adults. PLoS One. 2013;8(2):e55902.

47. Reiman EM, Chen K, Caselli RJ, Alexander GE, Bandy D, Adamson JL, et al. Cholesterol-related genetic risk scores are associated with hypometabolism in Alzheimer's-affected brain regions. Neuroimage. 2008;40(3):1214-21

\section{Figures}

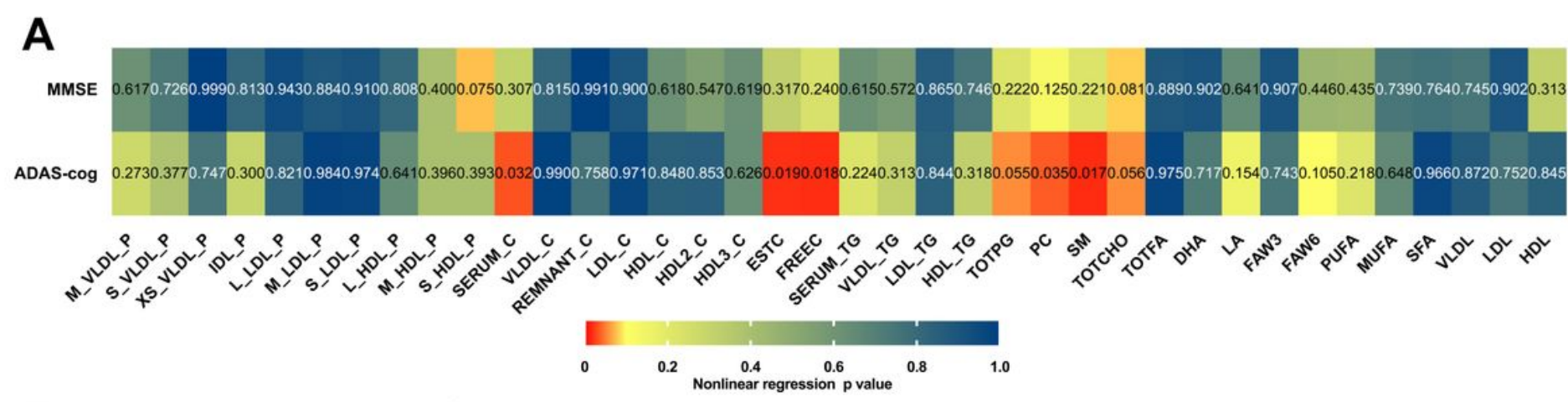

B
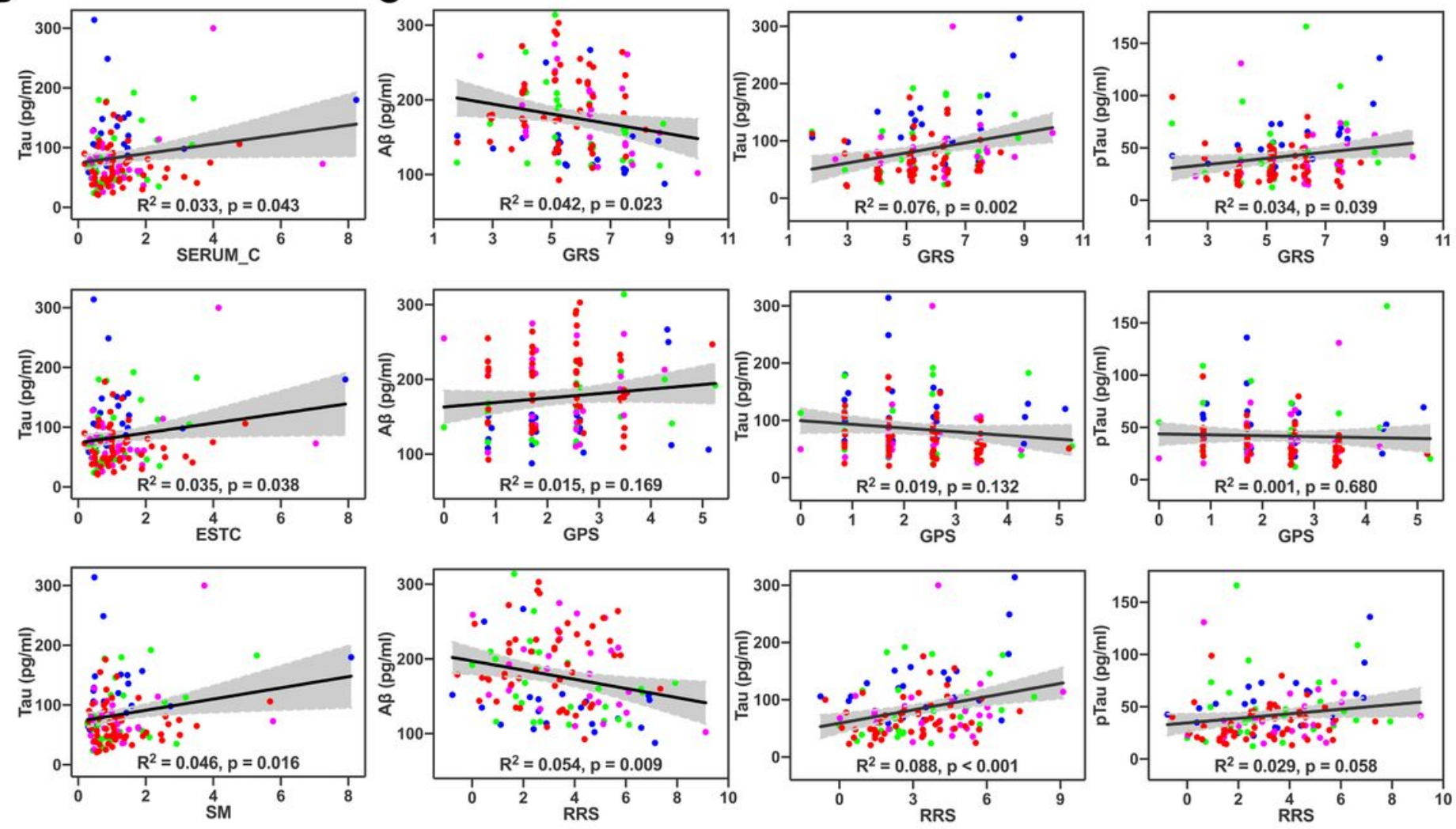

\section{Figure 1}

Regression analyses between polygenic scores, cerebrospinal fluid biomarkers, plasma cholesterol metabolites, and general cognition in the AD spectrum. A: Nonlinear correlations were plotted in heat map between blood cholesterol metabolites and cognitive scores. The color bar indicated nonlinear regression 
$p$ values ranging from 0 to 1 . Two-tailed $p$ values $<0.1$ were considered significant. B: Significant linear correlations were found between three cholesterol metabolism related markers in the blood and Tau level of cerebrospinal fluid in the AD spectrum. C: Linear regression between polygenic scores and cerebrospinal fluid biomarkers revealed that GRS was significantly correlated with $A \beta, T a u$, and even $p$ Tau levels, in the contrast, GPS was not correlated with any of cerebrospinal fluid biomarkers; while relative risk scores (RRS = GRS - GPS) was significantly influenced the $A \beta$, Tau but not $p$-Tau levels in the AD spectrum. Grey bands indicated $95 \%$ confidence intervals and binomial nonlinear regressions were applied in Fig. 1B and 1C. Two-tailed p values $<0.1$ were considered significant in Fig. 1B and 1C. Abbreviations: VLDL, very low-density lipoprotein; IDL, intermediate density lipoprotein; $L D L$, low density lipoprotein; HDL, high density lipoprotein; M_VLDL_P, total lipids, phospholipids, total cholesterol, cholesterol esters, free cholesterol and triglycerides in medium VLDL particles; S_VLDL_P, total lipids, phospholipids, total cholesterol, cholesterol esters, free cholesterol and triglycerides in small VLDL particles; XS_VLDL_P, total lipids, phospholipids, total cholesterol, cholesterol esters, free cholesterol and triglycerides in very small VLDL particles; IDL_P, total lipids, phospholipids, total cholesterol, cholesterol esters, free cholesterol and triglycerides in IDL particles; L_LDL_P, total lipids, phospholipids, total cholesterol, cholesterol esters, free cholesterol and triglycerides in large LDL particles; M_LDL_P, total lipids, phospholipids, total cholesterol, cholesterol esters, free cholesterol and triglycerides in medium LDL particles; S_LDL_P, total lipids, phospholipids, total cholesterol, cholesterol esters, free cholesterol and triglycerides in small LDL particles; L_HDL_P, total lipids, phospholipids, total cholesterol, cholesterol esters, free cholesterol and triglycerides in large HDL particles; M_HDL_P, total lipids, phospholipids, total cholesterol, cholesterol esters, free cholesterol and triglycerides in medium HDL particles; S_HDL_P, total lipids, phospholipids, total cholesterol, cholesterol esters, free cholesterol and triglycerides in small HDL particles; SERUM_C, serum total cholesterol; VLDL_C, total cholesterol in VLDL; REMNANT_C, remnant cholesterol (non-HDL, non-LDL cholesterol); LDL_C, total cholesterol in LDL; HDL_C, total cholesterol in HDL; HDL2_C, total cholesterol in HDL2; HDL3_C, total cholesterol in HDL3; ESTC, esterified cholesterol; FREEC, free cholesterol; SERUM_TG, serum total triglycerides; VLDL_TG, triglycerides in VLDL; LDL_TG, Triglycerides in LDL; HDL_TG, triglycerides in HDL; TOTPG, total phosphoglycerides; PC, phosphatidylcholine and other cholines; SM, sphingomyelins; TOTCHO, total cholines; TOTFA, total fatty acids; DHA, docosahexaenoic acid; LA, linoleic acid; FAW3, omega-3 fatty acids; FAW6, omega-6 fatty acids; PUFA, polyunsaturated fatty acids; MUFA, monounsaturated fatty acids; SFA, saturated fatty acids. $A \beta$, amyloid 1 to 42 peptide; Tau, total tau; PTau, tau phosphorylated at the threonine 181 position; MMSE, Mini-mental state examination; ADAS-Cog, Alzheimer's Disease Assessment Scale- Cognitive subscale; GRS, genetic risk score including APOE; GPS, genetic protective score; RRS, relative risk score; SERUM_C, serum total cholesterol; ESTC, esterified cholesterol; FREEC, free cholesterol; PC, phosphatidylcholine; SM, sphingomyelins; TOTCHO, total cholines. 

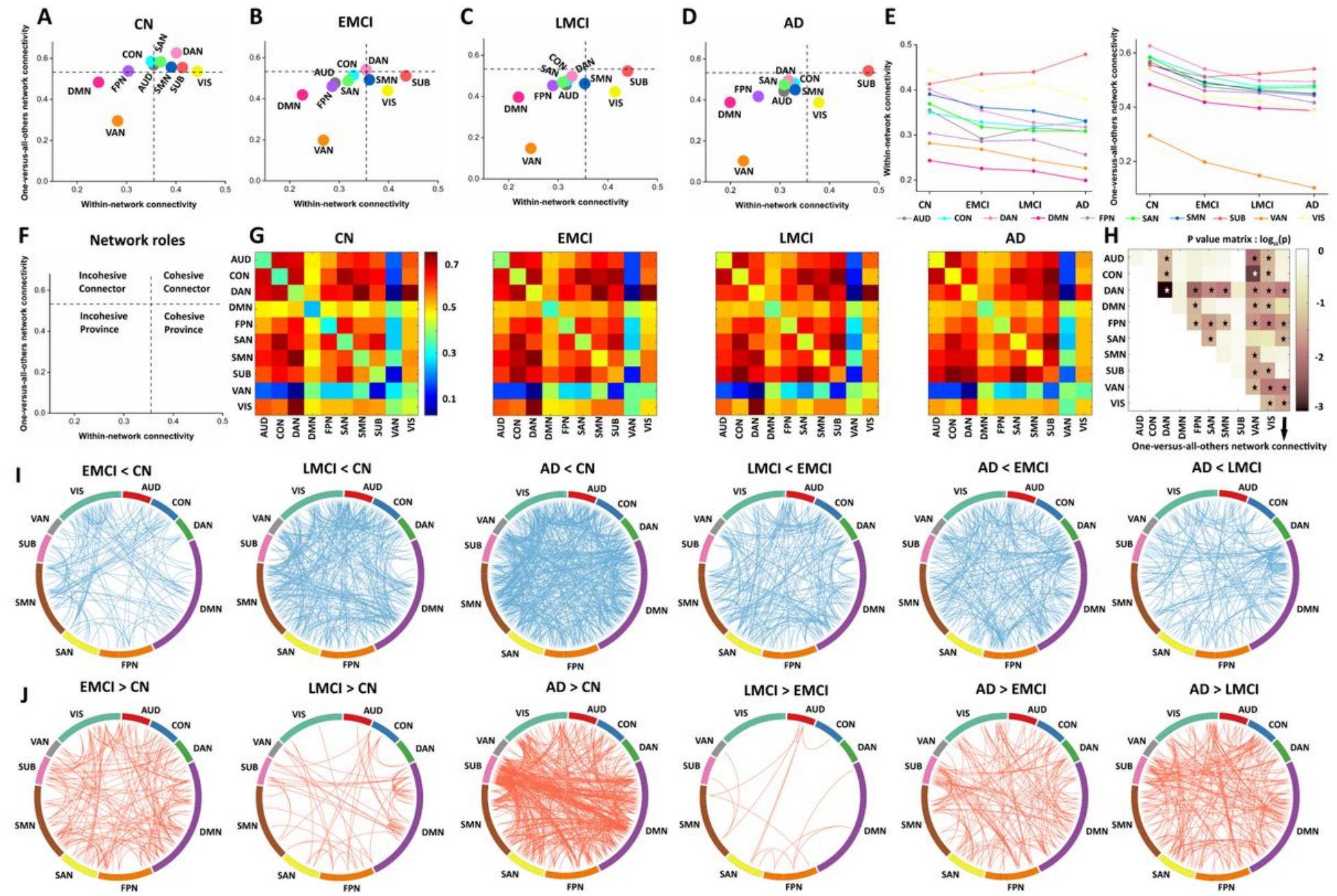

Figure 2

Network roles (F) in brain networks of CN (A), EMCl (B), LMCI (C), and AD (D); Dynamic trajectory of network role of large-scale RSNs within- and pairwise between-network connectivity matrices of the four groups $(\mathrm{G})$; A Line chart $(\mathrm{H})$ displays the dynamic trajectory of within- and one-versus-all-other network connectivity across the entire ADS; P value matrix of group differences in within-, one-versus-all-others-, and pairwise between-network connectivity in the ADS (F); Circos plot representation of significant grouplevel differences of neural connections among the ten RSNs in the ADS using the NBS method (I and J). Blue lines indicate decreased connectivity and red indicates increased connectivity in the ADS. Abbreviations: AUD, the auditory network; CON, the cingulo-opercular network; DAN, the dorsal attention network; DMN, the default mode network; FPN, the fronto-parietal network; SAN, the salience network; SMN, the sensory network; SUB, the subcortical network; VAN, the ventral attention network; VIS, the visual network. 

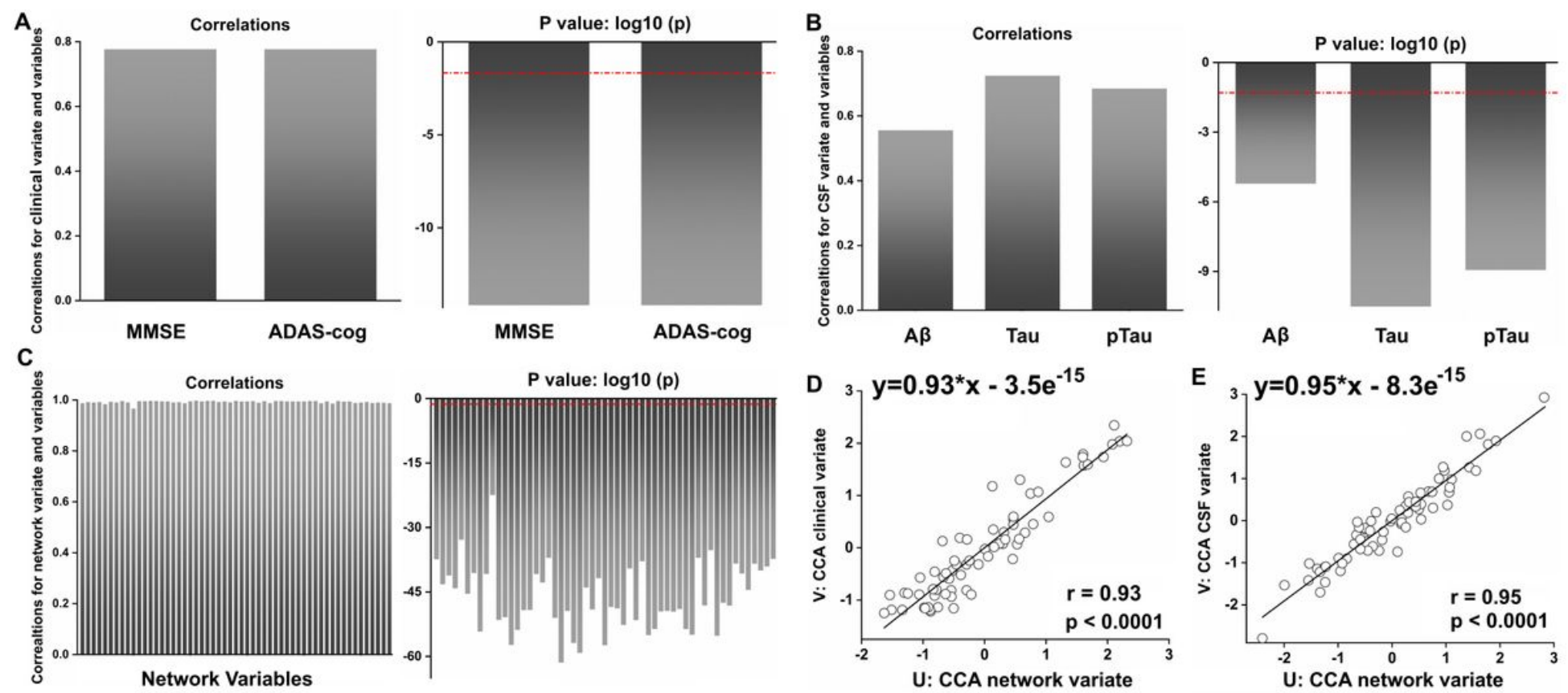

Figure 3

Correlations and their significance between the following in patients with $\mathrm{EMCl}, \mathrm{LMCl}$ and $\mathrm{AD}$ : the two clinical cognitive performance variables and clinical CCA mode (A); the three cerebrospinal fluid biomarker variables and CSF CCA mode (B); fifty-five within and pairwise between-network variables and network CCA mode (C); first pairwise CCA mode (D), and second pairwise CCA mode (E). Note P values in $A, B$ and $C$, have been log10-transformed. Red dashed lines represent a log10-transformed $P$ value of 0.05. Abbreviations: MMSE, mini-mental state examination; ADAS-cog, Alzheimer's disease assessment scale-cognitive section; $A \beta$, amyloid 1 to 42 peptide; Tau, total tau; pTau, tau phosphorylated at the threonine 181 position; CSF, cerebrospinal fluid; CCA, canonical correlation analysis. 

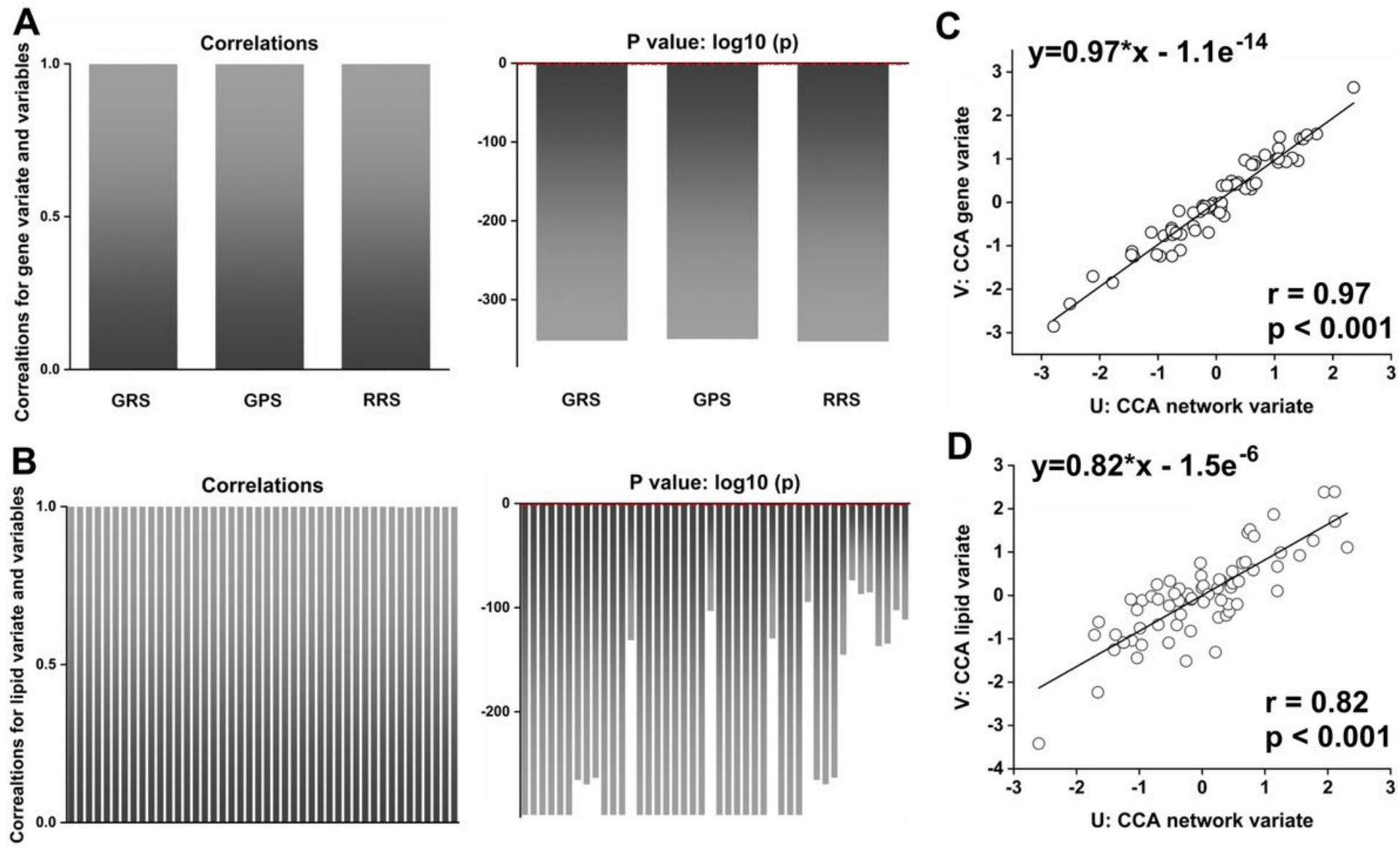

Figure 4

Correlations and their significance for patients with $\mathrm{EMCl}, \mathrm{LMCl}$ or $\mathrm{AD}$, for the following: $(\mathrm{A})$ three gene score variables and first gene CCA mode, (B) thirty-eight serum lipid variables and first level serum lipid CCA mode, (C) third pairs and (D) fourth pairs CCA mode. All data for lipid metabolites in blood were ztransformed. Note that $P$ values in $A$ and $B$ were log10-transformed. Red dashed lines represent $\log 10-$ transformed $P$ values of 0.05 . Abbreviations: GPS, genetic protective score; GRS, genetic risk score; RRS, relative risk score; CCA, canonical correlation analysis.
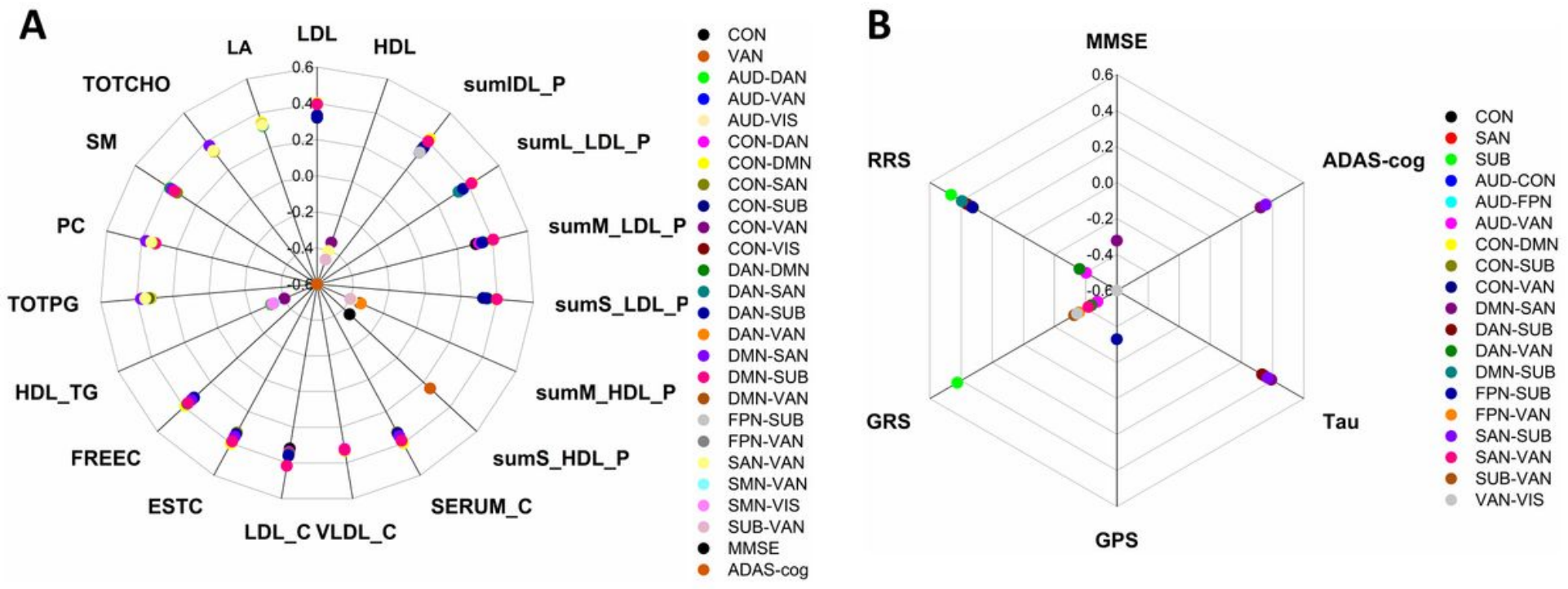


\section{Figure 5}

Radar plots indicating patterns of association for serum lipid, cognitive performance, CSF biomarkers, and polygenic scores to network connectivity. Values displayed by the dots in the radar plots are values of Pearson's correlation coefficients. All nodes represent a statistically significant correlation coefficient ( $p$ value<0.05). Abbreviations: AUD, the auditory network; CON, the cingulo-opercular network; DAN, the dorsal attention network; DMN, the default mode network; FPN, the fronto-parietal network; SAN, the salience network; SMN, the sensory network; SUB, the subcortical network; VAN, the ventral attention network; VIS, the visual network; LDL, low density lipoprotein; HDL, high density lipoprotein; sumIDL_P, sum of total lipids, phospholipids, total cholesterol, cholesterol esters, free cholesterol and triglycerides in IDL particles; sumL_LDL_P, sum of total lipids, phospholipids, total cholesterol, cholesterol esters, free cholesterol and triglycerides in large LDL particles; sumM_LDL_P, sum of total lipids, phospholipids, total cholesterol, cholesterol esters, free cholesterol and triglycerides in medium LDL particles; sumS_LDL_P, sum of total lipids, phospholipids, total cholesterol, cholesterol esters, free cholesterol and triglycerides in small LDL particles; sumM_HDL_P, total lipids, phospholipids, total cholesterol, cholesterol esters, free cholesterol and triglycerides in medium HDL particles; SERUM_C, serum total cholesterol; VLDL_C, total cholesterol in VLDL; LDL_C, total cholesterol in LDL; ESTC, esterified cholesterol; FREEC, free cholesterol; HDL_TG, triglycerides in HDL; TOTPG, total phosphoglycerides; PC, phosphatidylcholine and other cholines; SM, sphingomyelins; TOTCHO, total cholines; LA, linoleic acid; MMSE, mini-mental state examination; ADAS-cog, Alzheimer's disease assessment scale-cognitive section; Tau, total tau; GPS, genetic protective score; GRS, genetic risk score; RRS, relative risk score.

A

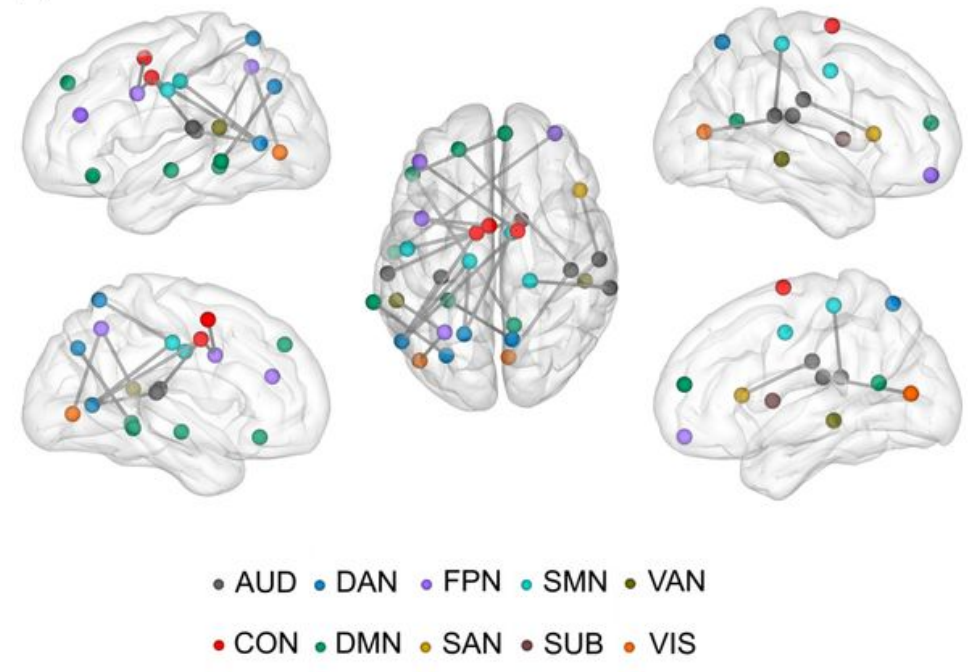

B

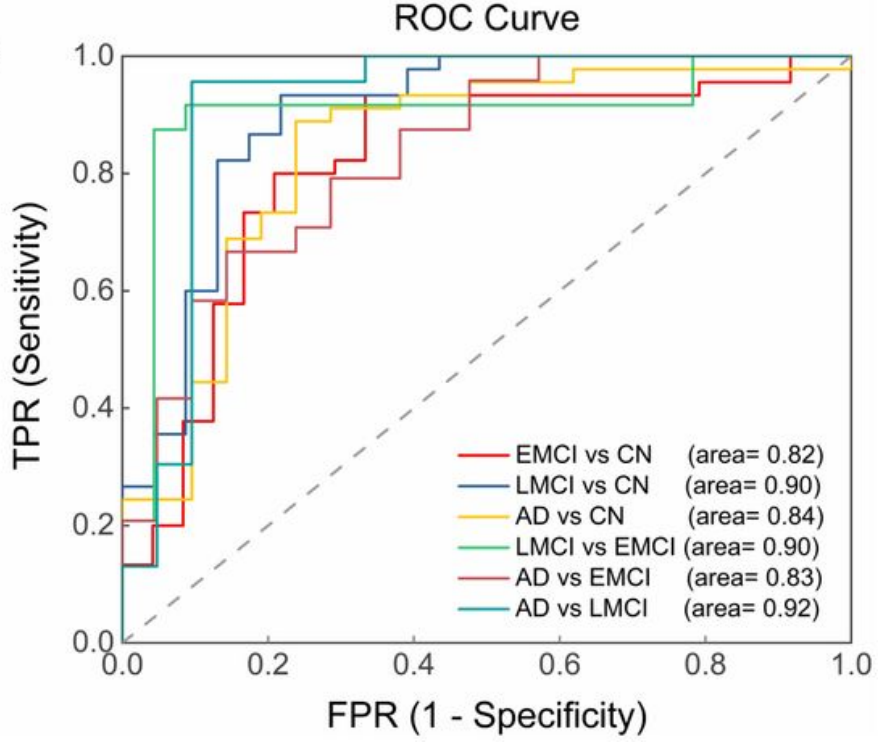

\section{Figure 6}

Lipid-associated imaging biomarker for classifying AD spectrum. Twenty-two functional connections were used for classification between groups. Node colors represent Power-atlas cortical and subcortical regions consisting of ten RSNs. B) Functional connections that showed group-level differences were used as the inputs for binary classification. All $p$ values of area under curve were $<0.001$. Abbreviations: AUD, 
the auditory network; CON, the cingulo-opercular network; DAN, the dorsal attention network; DMN, the default mode network; FPN, the fronto-parietal network; SAN, the salience network; SMN, the sensory network; SUB, the subcortical network; VAN, the ventral attention network; VIS, the visual network; ROC, receiver operating characteristic; TPR, true positive rate; $F P R$, false positive rate $\mathbb{C N}$, cognitively normal; $E M C l$, early mild cognitive impairment; $L M C l$, late mild cognitive impairment; $A D$, Alzheimer's disease.

\section{Supplementary Files}

This is a list of supplementary files associated with this preprint. Click to download.

- supplementaryart.docx 